\title{
Heuristic optimization of Bezier curves based trajectories for unconventional airships docking
}

\author{
Alessandro Ceruti \\ DIN Department of Industrial Engineering, University of Bologna, Bologna, Italy, (corresponding author: \\ alessandro.ceruti@unibo.it ) \\ Pier Marzocca \\ School of Engineering, Aerospace Engineering and Aviation Discipline, RMIT, Melbourne, Australia \\ Published in Aircraft Engineering and Aerospace Technology \\ DOI: https://doi.org/10.1108/AEAT-11-2014-0200
}

Please cite as: Ceruti, A. and Marzocca, P. (2017), "Heuristic optimization of Bezier curves based trajectories for unconventional airships docking", Aircraft Engineering and Aerospace Technology, Vol. 89 No. 1, pp. 76-86. https://doi.org/10.1108/AEAT-11-2014-0200

Deposit License: This article is ( $\odot$ Emerald Publishing Limited and permission has been granted for this version to appear here (IRIS - University of Bologna, Italy (https://cris.unibo.it/ ) ). Emerald does not grant permission for this article to be further copied/distributed or hosted elsewhere without express permission from Emerald Publishing Limited.] 


\title{
Final peer-reviewed manuscript of: Ceruti, A. and Marzocca, P. (2017), "Heuristic optimization of Bezier curves based trajectories for unconventional airships docking", Aircraft Engineering and Aerospace Technology, Vol. 89 No. 1, pp. 76-86.
}

\begin{abstract}
Purpose - This paper aims to describe a methodology to optimize the trajectory of unconventional airship performing a high-altitude docking manoeuvre.
\end{abstract}

Design/methodology/approach - The trajectories are based upon Bezier curves whose control points positions are optimized through Particle Swarm Optimization algorithm. A minimum energy strategy is implemented by considering the airship physical properties. The paper describes the mathematical model of the airships, the trajectories modelling through Bezier's curves and the optimization framework. A series of test cases have been developed to evaluate the proposed methodology.

Findings - Results obtained show that the implemented procedure is able to optimize the airship trajectories and to support their in-flight docking; a strong influence of the wind speed and course on the trajectories planning is highlighted.

Research limitations/implications - The wind speed considered in these simulations depends only on altitude and gusts effect has been neglected.

Practical implications - The proposed model can support the study of unconventional airship trajectories and to evaluate best in-air docking strategies.

Originality/value - The paper addresses the problem of trajectory optimization for a class of new air vehicles with an heuristic approach.

Keywords Airship, Bezier curves, Green Transportation, Heuristic algorithms, Trajectories optimization.

Paper type Research paper

\section{Introduction}

The interest in High Altitude Platforms (HAPs) is growing (Stockbridge et al., 2012) due to the ability of using solar energy, instead of fossil fuels, to power the air-vehicles, the reduction in weights obtained with composite and new materials, the improvements in control systems and inertial/GPS navigation, just to name but a few advancements aerospace and aviation have seen in this last decade. The use of photovoltaic films is particularly useful since it leads to a reduction of pollutant emissions in atmosphere and allows long range and endurance missions. Consequently, the drawbacks typical 
of the conventional airship (Khoury and Gillet, 1999) are reduced leading to more competitive air-vehicle solutions when a long mission with low carbon emissions requirements is planned. Recent studies focus the attention on the evaluation of unconventional airships for passenger transport (Dumas et al., 2011), (Rademacker, 2014). In particular, Dumas et al. (2011) proposes a transport modality for goods and people based on a novel architecture using two airship typologies: a cruiser and a feeder (Ilieva et al., 2014). The feeder airship can be considered as a sort of "vertical air lifter", similar in dynamics to an hot air balloon (Ellenrieder, 1999), connecting a cruiser airship loitering in the high atmosphere with on ground airports and facilities: in this way the cruiser does not change altitude to carry on or get off the payload (goods, people, etc). Detail on the possible propulsion of these airships can be found for instance in (Smith et al., 2013). This is useful since the smaller feeders can operate from small airports or complex suitable for airship docking, and the larger cruiser does not need to land and lift at each stop. This methodology requires a sort of "in air docking" to transfer payload from the feeder to the cruiser and vice-versa, so that this aspect has to be well investigated. A bulk of literature is available on the topic of solar airship design, control, trajectory planning, and aerodynamics. For instance, (Khoury and Gillet, 1999) is a comprehensive reference for traditional airships design and system description. One of the most recent monographs dealing with both traditional and high altitude airship by (Carichner and Nicolai, 2013) discusses methods for rapid airship sizing. The conceptual design of solar airships is detailed in Ceruti and Marzocca (2014) where unconventional configurations are considered, in Yu and Lu (2012) where trade-off analysis are presented around several design points, in Chen et al. (2011) providing a parametric analysis, and in Ceruti et al. (2014) where unconventional configurations are optimized through heuristic algorithms. Structural aspects related to the conventional and unconventional airships have been discussed in Liao and Pasternack, (2009) while an analysis dealing with control aspects has been presented in Yuwen et al. (2011). Strategies for airships trajectory control and optimization have been discussed in a variety of publications, noteworthy are the recent works presented in Guo and Zhu (2013) and in Lee et al. (2014). Additionally, an extensive study on the airship trajectory optimization has been carried out by Mueller et al. (2009a, 2009b), where trajectories can be drawn in space to obtain a minimum ascent time, a minimum energy consumption or a mix of them. In these studies, SNOPT algorithms (Murray et al., 2002) have been applied to find the set of control variables to identify optimal paths. Alternatively, other optimization methods can be applied in trajectory definition (Betts, 1998). Heuristic algorithms have also been applied to the trajectory optimization. For example, using heuristic methods Blasi et al. (2013) identified minimum distance paths to avoid no-fly zone areas.

In this paper, heuristic algorithms will be exploited to set the control points of trajectories based on the Bezier curves. A motivating factor is that such an approach can be used to perform the docking between two airships: the advantages over methods based on SNOPT and Sequential Programming Tools lie in the fact that these stretegie are suited for a moderate number of degrees of freedom (Gil et al. 2005). Also, classical control techniques like backstepping (Lee, 2014) 
are quite complex to implement, and computational performances decrease with the degrees of freedom of the problem (corresponding to the number of steps in which the trajectory is divided). The rest of the paper is organized as follows. After the introduction, notes on Bezier's curves and on the point mass airship dynamic model used in this work will be presented. It will be followed by a brief description of the Particle Swarm Optimization algorithm (Kennedy and Eberhart, 1995) used in this work, together with notes on the parameters to optimize, the Fitness function, and the optimization mathematical framework proposed. Finally, a section with a case study and a conclusion section are offered.

\section{Modelling of the Docking}

This section provides a description of the mathematical tools used to describe the trajectory and to carry on the simulations of the airship dynamics.

\section{Bezier Curve}

The Bezier curves have been introduced in 1962 to model smooth lines and surfaces to be used the design of cars, ships, and aircrafts (Mortenson, 1999). The success and spreading of these curves is mainly related to the fact that the mathematics (Farin, 1990) required to describe these geometrics is simple and can be easily implemented in computers or CAD systems. The Bezier curves approximate a control polygon based on a series of control points, whose number can be defined by user: the curve passes through the first and the last point of the control polygon, and it is tangent to the first and last segment of the control polygon in the starting and ending point respectively. Moving a control point affects all curves: consequently, it is possible to define in an easy way the tangent of the curve at the tips, however it is quite complex to force the curve to pass through a point in space by changing the position of the intermediate control points. The implementation of Bezier curves followed in this paper to model trajectories is based on the formula:

$$
B(t)=\sum_{i=0}^{n} b_{i, n}(t) P_{i}, \quad \text { with } \quad t \in[0 \div 1]
$$

Herein, $B(t)$ represent a three-dimensional column vector containing the $x, y, z$ position of the point in the space; $t$ is a parameter crossing along the curve, it is equal to 0 at its starting point, and to 1 at its ending point; $P_{i}$ represents a column vector containing the position in space of the $i$-th control point, $n$ is the degree of the curve, and the number of control points is $n+1$. The term $b_{i, n}(t)$ is called Bernstein basis polynomial of degree $n$ and can be computed through:

$$
b_{i, n}(t)=\left(\begin{array}{c}
n \\
i
\end{array}\right) t^{i}(1-t)^{n-i}, \quad \text { with } \quad i=0,1, \ldots, n-1, n
$$


Figure 1 shows an example of Bezier curve of degree $n=4$ in the plane: the control polygon is identified by the segments between the five control points $P_{0}, P_{1}, P_{2}, P_{3}, P_{4}$.

Figure 1 Bezier curve of degree 4.

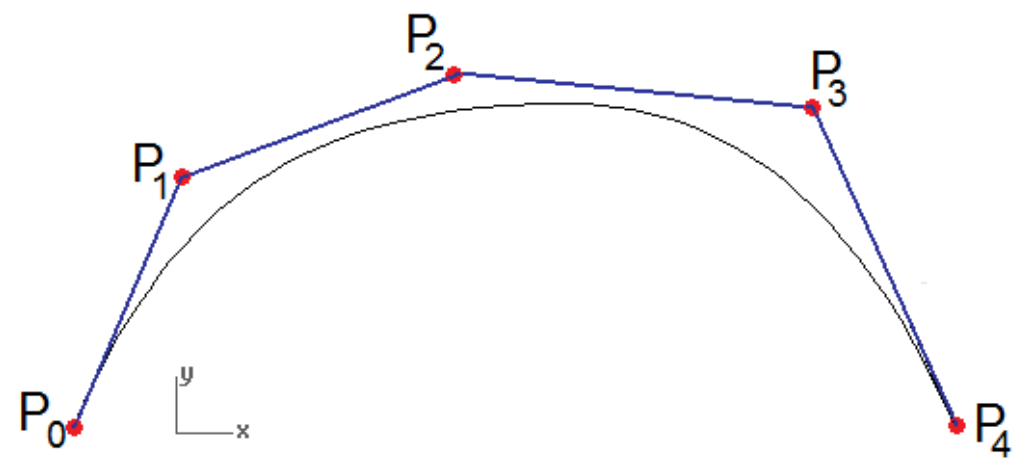

\section{Trajectories modelling}

Going to the cruiser/feeder transport airship concept discussed in the introduction, their trajectories will be modelled through 3D Bezier curves of degree 4, so that the definition of the position of 5 control points will be required. It is also worth noting that the property of the Bezier curves to be tangent to the first and last segment of the control polygon in the starting and ending point respectively is critical for the trajectories features definition.

In a typical docking scenario, one airship is on ground (the feeder), while the other one is cruising at high altitude (the cruiser): the engagement is considered successful if both the airships reach the same point at altitude with the same speed and direction. The problem to solve is to draw in the space two trajectories tangent each other at the docking point. It is supposed to know the starting point of both the cruiser $\left(P c_{0}=\left[X c_{\text {start, }}, Y c_{\text {start, }}, Z c_{\text {start }}\right]\right)$ and the feeder $\left(P f_{0}=\left[X f_{\text {start, }}, Y f_{\text {start }}\right.\right.$, $\left.Z f_{\text {start }}\right)$, and their initial course $\psi_{c}$ and $\psi_{f}$ (measured respect to the North), as well as the docking altitude $\left(Z_{\text {dock }}\right)$. Figure 2 shows the trajectory of the feeder and cruiser modelled through a 4 degrees Bezier curve, so that 4 additional points are necessary for each trajectory to model the track, assuming that the first point is known. Distances are expressed in meters in North, East, and Up direction respect to the origin, represented by the feeder take off point.

Figure 2 Examples of trajectories of the feeder (left) and cruiser (right) with control polygon. 

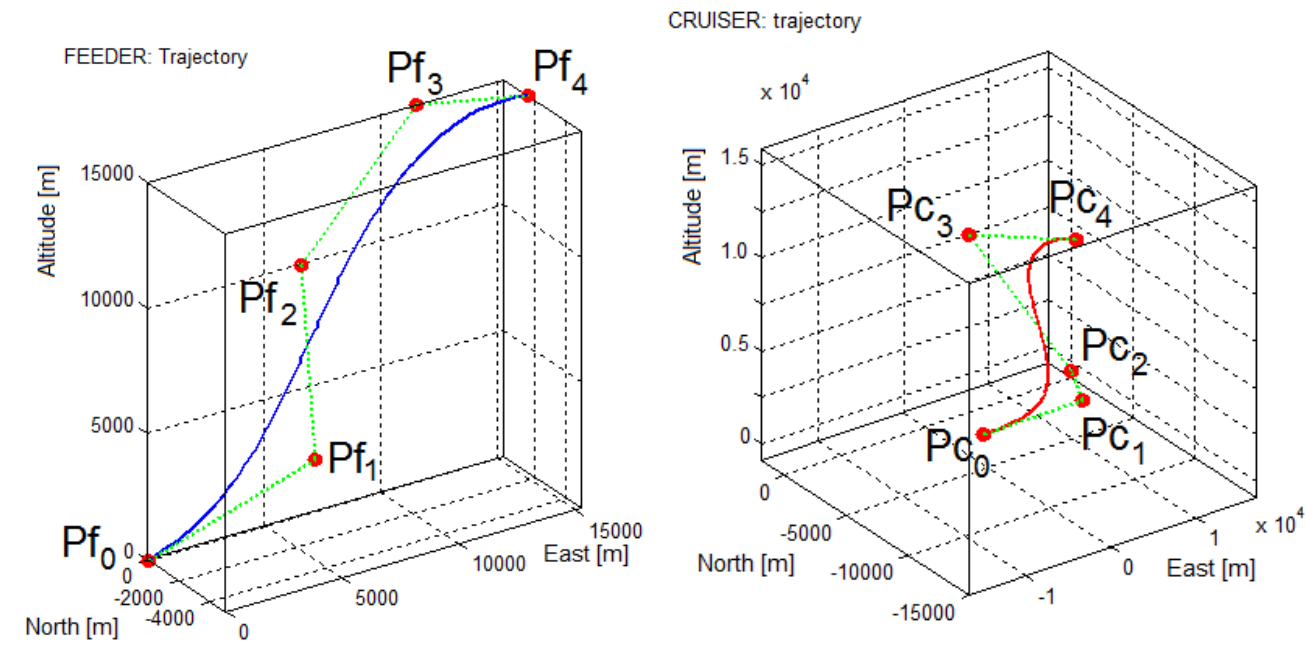

To guarantee a degree of convergence and physical plausibility, the following simplifications are applied to the feeder:

$$
\begin{gathered}
P f_{0}=\left[X f_{\text {start }} ; Y f_{\text {start }} ; 0\right] \\
P f_{1}=\left[k_{1} \sin \psi_{f} ; k_{1} \cos \psi_{f} ; Z_{\text {dock }} / 3\right] \\
P f_{2}=P f_{1}+\left[\Delta x f_{2} ; \Delta \mathrm{yf}_{2} ; 2 Z_{\text {dock }} / 3\right] \\
P f_{3}=P f_{2}+\left[\Delta x f_{3} ; \Delta \mathrm{yf}_{3} ; Z_{\text {dock }}\right] \\
P f_{4}=P f_{3}+\left[\Delta x f_{4} ; \Delta \mathrm{yf}_{4} ; 0\right]
\end{gathered}
$$

The trajectory of the feeder is known once the 7 parameters $k_{1}, \Delta x f_{2}, \Delta y f_{2}, \Delta x f_{3}, \Delta y f_{3}, \Delta x f_{4}, \Delta y f_{4}$ have been defined. For the cruiser the following constraints are adopted:

$$
\begin{gathered}
P c_{0}=\left[X c_{\text {start }} ; Y c_{\text {start }} ; Z_{\text {dock }}\right] \\
P c_{1}=P c_{0}+\left[k_{2} \operatorname{dist}\left(P c_{0}, P c_{4}\right) \sin \psi_{c} ; k_{2} \operatorname{dist}\left(P c_{0}, P c_{4}\right) \cos \psi_{c} ; Z_{\text {dock }}\right] \\
P c_{4}=P f_{4} \\
P c_{3}=P f_{4}-k_{3}\left(P f_{4}-P f_{3}\right) \\
P c_{2}=0.5\left(P c_{1}+P c_{3}\right)+\left[\Delta x c_{2} ; \Delta c_{2} ; 0\right]
\end{gathered}
$$

In this case 4 parameters are necessary to define the cruiser trajectory: $k_{2}, k_{3}, \Delta x c_{2}, \Delta y c_{2}$ since all the other data can be computed or have been assumed as defined by the user. It is worth noting that with this definition, and thanks to the properties of the Bezier's curves, the two trajectory are tangent each other at the ending point, as figure 3 shows.

Figure 3 Example of trajectories of the feeder and cruiser (top view). 


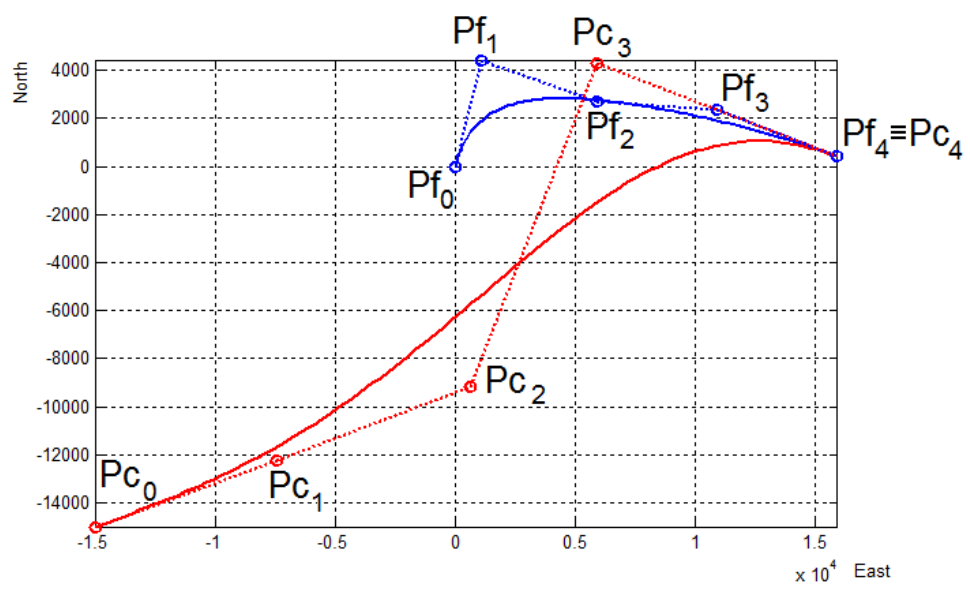

\section{Trajectories modelling}

The mathematical model of the airship dynamics selected for this study assumes that the airships can be represented by their mass point, as described in (Mueller et al., 2009b). The following equations apply:

$$
\begin{gathered}
\dot{V}=\frac{(B-W) \sin \gamma+T \cos (\alpha+\mu)-D-\text { Vol } \dot{\rho} V}{m+m_{a x}}-\dot{W}_{x} \\
\dot{\gamma}=\frac{(B-W) \cos \gamma+T \sin (\alpha+\mu)+L}{\left(m+m_{a z}\right) V}+\frac{\dot{W}_{z}}{V} \\
\dot{\psi}=\frac{F_{\text {lat }}}{\left(m+m_{\text {ay }}\right) V \cos \gamma}-\frac{\dot{W}_{y}}{V \cos \gamma}
\end{gathered}
$$

where $V$ is the speed of the airship along the trajectory, $\gamma$ is the trajectory angle (defined as the vertical angle between the segment tangent to the trajectory and a plane tangent to the earth surface), $\psi$ is the course (respect to the North), $B$ and W buoyancy and weight, $D$ and $T$ are drag and thrust, $L$ stands for lift, $F_{\text {lat }}$ is a lateral force obtained with control surfaces or thrust system, $\alpha$ is the angle of attack (AoA), $\mu$ is defined as the angle between the reference line of the airship (the line used to measure the $\mathrm{AoA}$ ) and the propulsion system thrust, $\mathrm{Vol}$ is the airship volume and $\rho$ the air density at current altitude. The airship mass and the added masses (Tuveri et al., 2014) along $x, y, z$ axis can be expressed respectively with: $m, m_{a x}, m_{a y}, m_{a z}$. Finally, $W_{x}, W_{y}, W_{z}$ represents the projection of the wind speed in $x, y, z$ body axis.

Herein, the displacements of the airship in a reference system with origin $O$ on the ground, $X$ and $Y$ axis lying in a plane tangent to the Earth surface in the Origin $0, X$ axis pointing East, $Y$ towards the North and $Z$ upwards and perpendicular to the plane can be computed through:

$$
\begin{gathered}
\Delta \dot{X}=V \cos \gamma \sin \psi+W_{E a s t} \\
\Delta \dot{Y}=V \cos \gamma \cos \psi+W_{N o r t h} \\
\Delta \dot{Z}=V \sin \gamma+W_{u p}
\end{gathered}
$$


$W_{\text {East }}, W_{\text {North }}, W_{u p}$ are the components of the wind speed along the North, East and Up direction. The displacements can be expressed also in terms of latitude (Lat), longitude (Long), and altitude $(h)$ by the following set of equations, in which $R$ represents the Earth mean Radius:

$$
\begin{aligned}
\text { Long } & =\frac{\Delta \dot{X}}{(R+h) \cos \lambda} \\
\text { L } \dot{a} t & =\frac{\Delta \dot{Y}}{(R+h)} \\
\dot{h} & =\Delta \dot{Z}
\end{aligned}
$$

Finally, the terms in Eq. (3) related to the wind speed can be computed from:

$$
\begin{aligned}
& \dot{W}_{x}=\dot{W}_{\text {East }} \cos \gamma \sin \psi+\dot{W}_{\text {North }} \cos \gamma \cos \psi \\
& \dot{W}_{y}=\dot{W}_{\text {East }} \cos \psi-\dot{W}_{\text {North }} \sin \psi \\
& \dot{W}_{z}=\dot{W}_{\text {East }} \sin \gamma \sin \psi+\dot{W}_{\text {North }} \sin \gamma \cos \psi
\end{aligned}
$$

This mathematical model can be applied to model the cruiser trajectory. However, if a feeder with a dynamics similar to that of a hot air balloon (Ellenrieder, 1999) is considered, it is difficult to apply the above scheme since the trajectory angle can be close to the vertical and the airship lies horizontal, so that a large negative angle of attack would appear and numerical instabilities can arise; moreover, it is assumed that the airship does not change its tilt respect to the horizon. Consequently, in this case a new set of equations, in which the lateral and horizontal dynamics has been decoupled, can be used. This simplification can be considered quite crude, from a mathematical and aerodynamics point of view can be presented as:

$$
\begin{gathered}
\dot{V}_{\text {hor }}=\frac{T_{\text {long }}-D_{\text {hor }}}{m+m_{\text {ax }}}-\dot{W}_{x} \\
\dot{V}_{\text {vert }}=\frac{(B-W)+L-D_{\text {vert }}}{\left(m+m_{a z}\right)}+\dot{W}_{z} \\
\dot{\psi}=\frac{T_{\text {lat }}}{\left(m+m_{\text {ay }}\right) V_{\text {hor }}}-\frac{\dot{W}_{y}}{V_{\text {hor }}}
\end{gathered}
$$

where $D_{\text {hor }}$ is the drag of the airship when a horizontal relative speed $\left(V_{\text {hor }}\right)$ is considered, $D_{\text {vert }}$ is the drag of the airship when a vertical relative speed $\left(V_{\text {vert }}\right)$ is considered, while $T_{\text {long }}$ and $T_{\text {lat }}$ represent thrust in longitudinal and lateral direction. The lift $L$ is the vertical force which is obtained when there is an horizontal relative speed $\left(V_{\text {hor }}\right)$ between the wind and the airship. With the above simplification, the following applies: 


$$
\begin{gathered}
\Delta \dot{X}=V_{\text {hor }} \sin \psi+W_{\text {East }} \\
\Delta \dot{Y}=V_{\text {hor }} \cos \psi+W_{\text {North }} \\
\Delta \dot{Z}=V_{\text {vert }}+W_{\text {up }}
\end{gathered}
$$

Obviously, if a feeder with a prevalent horizontal motion is considered (low trajectory angle), the equations selected for the cruiser applies.

\section{Optimization framework}

This section provides an implementation of the model presented in the earlier section with the application to the optimization of the cruiser/feeder trajectories.

\section{Dynamics along the trajectories}

The trajectory of the cruiser is modelled in two segments: a first one in which the cruising speed $V_{\text {cruise }}$ (relative to the wind) is followed along the trajectory, and a second one in which the speed is increased or decreased up to the docking speed $V_{\text {dock, }}$ which is reached at the end of the path. The trajectory is discretized in $N$ points, so that for each couple of consecutive points $\Delta X, \Delta Y, \Delta Z$ can be computed. It is also possible to compute the time it takes to cover the distance between two consecutive points $(\Delta t)$. The set of equations $(4 \mathrm{a}, \mathrm{b}, \mathrm{c})$ can be written as:

$$
\begin{gathered}
\frac{\Delta \mathrm{X}}{\Delta \mathrm{t}}=V \cos \gamma \sin \psi+W_{\text {East }} \\
\frac{\Delta \mathrm{Y}}{\Delta \mathrm{t}}=V \cos \gamma \cos \psi+W_{\text {North }} \\
\frac{\Delta \mathrm{Z}}{\Delta \mathrm{t}}=V \sin \gamma+W_{u p}
\end{gathered}
$$

where $\Delta t, \gamma, \psi$ are unknown variables which can be computed solving the system of three Eqs. $(9 a, b, c)$ when $V, W_{\text {East }}$, $W_{\text {North }}, W_{\text {up }}$ are known. Now it is possible also to compute the terms $(B-W)$ and $T$ from Eqs. $(3 a, b)$ since all the parameters are known, except for $\alpha$ and $\mu$. The latter are related to the airship features and can be set equal to zero as a first approximation. The terms $D$ and $L$ can be computed with:

$$
\begin{aligned}
D & =\frac{1}{2} \rho C_{D} V_{o l^{2 / 3}} V^{2} \\
L & =\frac{1}{2} \rho C_{L} V_{o l}^{2 / 3} V^{2}
\end{aligned}
$$


while Eq. (3c) can be used to compute the lateral force $F_{\text {lat. }}$ The trajectory of the feeder is divided into three segments: vertical acceleration, climb with constant vertical speed, deceleration to zero vertical speed. The vertical climb speed is selected keeping into consideration the needs of comfort by the passengers and the limits in vertical force: it is in fact assumed that the vertical speed can be achieved only with a change of the $(B-W)$ term. During the first segment, at first the unknown term $\Delta t$ can be computed with the Eq. (11a), while in the following $\psi, V_{\text {hor }}$ can be computed solving the system of Eq. $(11 b, c)$ :

$$
\begin{gathered}
\Delta \mathrm{t}=\frac{\Delta \mathrm{Z}}{\left(V_{\text {vert }}+W_{\text {up }}\right)} \\
\operatorname{tg} \psi=\frac{\frac{\Delta \mathrm{X}}{\Delta \mathrm{t}}-W_{\text {East }}}{\frac{\Delta \mathrm{Y}}{\Delta \mathrm{t}}-W_{\text {North }}} \\
V_{\text {hor }}=\frac{\left(\frac{\Delta \mathrm{Y}}{\Delta \mathrm{t}}-W_{\text {North }}\right)}{\cos \psi}
\end{gathered}
$$

The vertical velocity $\left(V_{\text {vert }}\right)$ is increased along a defined number of points of the trajectory up to the value $V_{\text {climb. }}$ In the second segment of flight, a constant vertical speed is assumed and the equations (11a,b,c) can be applied, assuming for $V_{\text {vert }}$ the constant value $V_{\text {climb }}$. During the third path of the trajectory, the horizontal speed should reach a value $V_{\text {dock, }}$ which can be optimized to obtain feasible trajectories for cruiser and feeder. In this case, a different set of equation must be used to compute the value of $\Delta \mathrm{t}, \psi, V_{\text {hor }}, V_{\text {vert }}$ along this part of trajectory. The horizontal speed $V_{\text {hor }}$ is assumed to linearly increase/decrease from the final speed computed in the second segment of the trajectory up to the $V_{\text {docking }}$ speed. The parameters to be computed $\psi, \Delta t$, can be found solving the system of equations $12 \mathrm{a}, \mathrm{b}$ :

$$
\begin{gathered}
\frac{\Delta \mathrm{X}}{\Delta \mathrm{t}}=V_{\text {hor }} \sin \psi+W_{\text {East }} \\
\frac{\Delta \mathrm{Y}}{\Delta \mathrm{t}}=V_{\text {hor }} \cos \psi+W_{\text {North }}
\end{gathered}
$$

Finally, the vertical speed is computed with the Eq. (13):

$$
V_{\text {vert }}=\frac{\Delta \mathrm{Z}}{\Delta \mathrm{t}}-W_{u p}
$$

Once $V_{\text {hor }}, V_{\text {vert }}, \psi, \Delta t$ are known for each point of the feeder trajectory, the values of $T_{\text {long }},(B-W), T_{\text {lat }}$ can be computed using the set of Eqs. $(7 \mathrm{a}, \mathrm{b}, \mathrm{c})$ and obtaining $D_{\text {hor }}, D_{\text {vert }} L$ as: 


$$
\begin{gathered}
D_{\text {hor }}=\frac{1}{2} \rho C_{\text {Dhor }} A_{\text {lat }} V_{\text {hor }}{ }^{2} \\
D_{\text {vert }}=\frac{1}{2} \rho C_{\text {Dvert }} A_{\text {vert }} V_{\text {vert }}^{2} \\
L=\frac{1}{2} \rho C_{L} A_{\text {lat }} V_{\text {hor }}{ }^{2}
\end{gathered}
$$

Herein $A_{\text {lat }}$ is the projection of the airship shape on the lateral plane (and it is used in the horizontal dynamics) and $A_{v e r t}$ is the projection on the horizontal plane (and is used for the vertical dynamics). Once the points of the cruiser/feeder trajectories and the main features of the airships have been defined, it is possible to evaluate along the trajectory the longitudinal thrust, the lateral force, the course, the vertical and horizontal speeds, the difference between buoyancy and weight, and the time needed by the cruiser/feeder to follow the trajectory. By increasing the number of points in which the Bezier curves is discretized, precision with higher level of confidence is reached. It is worth to note that the computational weight linearly increases with the number of points in which the trajectory is divided $(\mathrm{N})$; on the other hand, the optimization is carried out on the position of the Bezier Curves control points position. As said before, due to the properties of the Bezier Curves it is simple to respect constraints like the tangency at the docking point with a reduced number of control points. Accordingly to the physics of the problems, in case of front wind or combination of the values of $\Delta \mathrm{X}$ and $\Delta \mathrm{Y}$ there is no solution for the system of Eqs. 8 and 12. In this case, the value of the unknown parameters is kept equal to that of the previous step, but a flag value $\left(N_{u n f}\right)$ is increased by one each time this happen. The value $N_{u n f}$ is introduced in the Fitness function (both for cruiser and feeder) to penalize the solution so that during the optimization the PSO avoids unfeasible trajectories.

\section{Particle Swarm Optimization}

To optimize (Yang, 2005) a problem means to find a set of values able to minimize or maximize a function, called Fitness function or Target function. Operative research, gradient or heuristic methods can be exploited to search the optimal set of parameters in a shorter time than what possible with a Monte Carlo (Fishman, 1995) strategy based on a purely random search. The heuristic algorithms are strategy driven procedures which typically imitates nature or efficient dynamics to improve the computational time and efficiency. Among others Genetic Algorithms (Goldberg, 1989), Particle Swarm Optimization (PSO) (Kennedy and Eberhart, 1995), Differential Evolution (Storn and Price, 1997), Imperialist Competitive Algorithms (Atashpaz-Gargari and Lucas, 2007), Gravitational Search (Hsiao et al., 2005), Water Drops [Duan et al., 2008], Tabu Search (Yang, 2005) are algorithms commonly used to solve industrial (Tornabene and Ceruti, 2013) and aerospace engineering problems (Ceruti et al., 2014). In this paper, the PSO algorithm will be applied, since good results have been obtained on trajectory optimization in (Blasi et al., 2013). This algorithm has been introduced for the first time by Kennedy (Kennedy and Eberhart, 1995), but there have been many further studies proposing variations and slight 
changes on the original formulation to improve its efficiency. The original algorithm is based upon two recursive steps: the update of the particle speed and position. On the one hand, a solution to the problem in the $n$-dimensional space of the parameters can be translated into a position of a particle, while on the other hand the direction in the $n$-space toward a new position to explore can be represented by an $n$-dimensional speed. Following (Kennedy and Eberhart, 1995), the particle speed can be so expressed with:

$$
v_{i}(k+1)=\phi(h) v_{i}(k)+\alpha_{1}\left[\gamma_{1 i}\left(p_{i}-x_{i}(k)\right]+\alpha_{2}\left[\gamma_{2 i}\left(G-x_{i}(k)\right]\right.\right.
$$

where $i$ is the index of the single particle, $k$ the algorithm step, $\Phi(k)$ the inertia function, $v_{i}$ the velocity of the $i$-th particle, $\alpha_{1,2}$ the acceleration constants, $p$ the best position found by the $i$-th particle (called also personal best), $G$ the best position found by the whole swarm (it is the best position within the personal bests), while $\gamma_{1,2}$ is a random number in the interval $[0 \div 1]$.

The position of the particles is expressed as:

$$
x_{i}(k+1)=x_{i}(k)+v_{i}(k+1)
$$

where $x_{i}$ is the position of the $i$-th particle, and $v_{i}(k+1)$ is the updated velocity of the $i$-th particle.

Two strategies can be used for the termination of the simulation: in the first one it stops after a pre-set number of iterations, while another condition can be represented by stopping the iterations when no improvement in solution is achieved for a defined number of consecutive iterations. In this latter case, the position of all the particles collapses into a single point in the $n$-dimensional space, which is the optimal one.

\section{Two steps optimization}

The optimization of the problem is split in two consecutive optimizations; in the first one, the feeder paths is optimized, while, in the second, the cruiser trajectory is optimized and part of the results obtained in the feeder optimization are exploited. It is useful to split in two the optimization because in this way the computational time is reduced since a smaller number of parameters is optimized at once. Considering the feeder optimization, the parameters to set are: $k_{1}, \Delta x f_{2}, \Delta y f_{2}$, $\Delta x f_{3}, \Delta y f_{3}, \Delta x f_{4}, \Delta y f_{4}$, plus the docking speed $V_{\text {dock }}$ and the point (NF3) of the feeder trajectory in which the third segment starts. NF3 is expressed as NF3=round $\left(n f_{3 r d}{ }^{*} \mathrm{~N}\right)$, where $n f_{3 r d}$ is a \% value multiplied by $N$, that is, as already introduced, the total number of points in which the feeder trajectory is divided; round is the rounding to closest integer number 
function. A total of 9 parameters is set with the PSO optimization. The definition of the Fitness function is always a critical task: it expresses in a mathematical way the end-user requirements. For the feeder, it is critical to save energy as much as possible. This is related to the weight of the batteries to be stored on board, to reduce the peaks in thrust below the maximum capacity of the propulsion system, to avoid excessive values of the $(B-W) / W$ parameter. Consequently, the energy expended in each trajectory step in which the trajectory is divided, can be computed for feeder with:

$$
E n=\sum_{i=1}^{N-1} \sqrt{\left({T_{\text {long } i}}^{2}+{T_{\text {lat } i}}^{2}\right)} * \text { space }_{i}
$$

where $T_{\text {long } i}, T_{\text {lat } i}$, space $_{i}$ are the longitudinal and lateral thrust along the $i$-th step of the trajectory and the space covered in the $i$-th step. Considering a number of points $N$ computed along the trajectory, $N-1$ is the number of steps in which the trajectory is divided. The Fitness function to be minimized for the feeder can be expressed as:

FitnesS feeder $=E n^{*}\left(1+\left(k_{f f} N_{T m a x}+k f f_{2} N_{B}-w_{+}+k f f_{3} N_{\text {unf }}\right)\right)$

where $N_{T \max }$ is the number of steps in which the maximum longitudinal thrust $T_{\max }$ value is crossed, and $N_{B-W}$, is the number of trajectory steps in which $(B-W) / W$ excesses a preset ratio, $N_{\text {unf }}$ is the number of steps in which the solution of the system of Eq. 12 has no solution; $k f f_{1}, \mathrm{kff}_{1}, \mathrm{kff}_{1}$ are weight parameters which can be used to help the convergence towards a solution satisfying the constraints on maximum thrust, maximum buoyancy, and feasibility of the trajectory. The Fitness expression gives the priority to the zeroing of the number of steps in which the maximum values for the thrust and difference in buoyancy and trajectory feasibility are obtained respect to the minimization of the energy spent along the trajectory.

Once the optimization of the feeder trajectory has been carried out and the 9 design parameters have been set, it is possible to start the optimization of the cruiser trajectory. The P3 and P4 points of the feeder trajectory are necessary input for the computation of the cruiser trajectory, together with the optimized engagement speed $V_{\text {dock. }}$ The five parameters to optimize for the cruiser are $k_{2}, k_{3}, \Delta x c_{2}, \Delta y c_{2}$, NC2; NC2 is the point of the cruiser trajectory in which the second segment starts. It is described in a similar manner to $n f_{3 r d}$, so that also in this case we introduce the term $n c_{2 n d}$ representing the percentage of the cruiser trajectory total number of points corresponding to the point in which the second segment starts. The cruiser trajectory is assumed to present small changes in altitude, to avoid problems in buoyancy change. On the other hand, a problem could be represented by sharp changes in airship trajectory, where the lateral force should be considered. As already stated, the value of $N_{\text {Tmax }}$ is equal to the number of steps in which the value of the maximum cruiser longitudinal thrust (absolute value) is exceeded, and $N_{\text {Flat, }}$, is equal to the number of steps in which the 
lateral force (absolute value) along the trajectory exceeds the maximum allowable lateral thrust $\left(M_{a x L T}\right)$, and $N_{\text {unf }}$ is related to the number of steps in which the system of equations (8) has no solution. If vertical control surfaces are applied to the airship, the value of the lateral force can be reduced assuming that part of the lateral force can be obtained by deflecting surfaces. This contribution is proportional to the square of the speed. The cruiser Fitness function can be so computed as:

$$
\text { Fitness }_{\text {cruiser }}=E n^{*}\left(1+\left(k f c_{1} N_{\text {Tmax }+} k f c_{2} N_{\text {Flat }}+k f c_{3} N_{\text {unf }}\right)\right)
$$

As for the feeder, $k f c_{1}, k f c_{2}, k f c_{3}$ are weights which can be introduced to help convergence towards acceptable solutions, by increasing the term related to the constraints which is harder to respect.

Analogously to what presented in eq. (17), the propulsive energy expended by the cruiser $(E n)$ is obtained as:

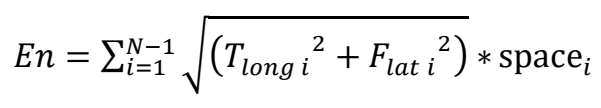

After the initial optimization of the feeder airship's parameters, another optimization is carried out for the cruiser, exploiting part of the results of the first optimization.

\section{Simulation scenario}

The optimization framework has been implemented and multiple simulation scenarios have been tested with several airship models; for sake of brevity, only an example will be included in this paper. The wind speed considered in these tests has been taken from Mueller et al. (2009b); in particular, in the following simulations the value of the spring season wind has been considered. The following Table 1 presents the features of the cruiser and feeder airships considered in the test herein included.

\section{Table 1 Cruiser and Feeder properties}

\begin{tabular}{llll}
\hline Feature & Cruiser & \multicolumn{2}{c}{ Feeder } \\
\hline \hline Altitude & $15 \mathrm{Km}$ & $0 \mathrm{Km}$ & $15 \mathrm{Km}$ \\
\hline Volume $\left(\mathrm{m}^{3}\right)$ & $2,760,000$ & 12,250 & 90,600 \\
\hline$C_{D}(-)$ & 0.04 & - & - \\
\hline$A_{\text {lat }}$ & - & 270 & 2100 \\
\hline Avert & - & 5000 & 5000 \\
\hline
\end{tabular}




\begin{tabular}{llll}
\hline CDhor $(-)$ & - & 0.31 & 0.32 \\
\hline$C_{\text {Dvert }}(-)$ & - & 1.36 & 1.16 \\
\hline$C_{L}(-)$ & 0 & 0.06 & -0.15 \\
\hline$T_{\max }(\mathrm{N})$ & 105,000 & 6,000 & 6,000 \\
\hline$M_{\text {axLT }}(\mathrm{N})$ & $0.333^{\star} T_{\max }$ & & \\
\hline$m_{a x}(\mathrm{~kg})$ & 59,000 & 480 & 3,300 \\
\hline$m_{a y}(\mathrm{~kg})$ & 204,000 & 460 & 3,350 \\
\hline$m_{a z}(\mathrm{~kg})$ & $1,300,000$ & 69,000 & 17,000 \\
\hline$((\mathrm{B}-\mathrm{W}) / \mathrm{W})_{\max }$ & & 0.5 & 0.2 \\
\hline
\end{tabular}

Both the trajectory of the cruiser and feeder has been divided in 100 points, so that there are 99 steps for cruiser and feeder trajectory. The following Table 2 includes the symbols and the ranges of change of the parameters to be optimized for the feeder; the last column presents also the optimized value. The range of parameters has been set following the experience of the authors and after some preliminarily test carried out to better understand the physics of the problem.

Table 2 Parameters to be optimized: feeder

\begin{tabular}{lllll}
\hline$\#$ & Parameter symbol & Minimum & Maximum & Optimized \\
\hline \hline 1 & $k_{1}(\mathrm{~m})$ & 1,000 & 5,000 & 1,000 \\
\hline 2 & $\Delta \mathrm{xf}_{2}(\mathrm{~m})$ & $-10,000$ & 10,000 & 10,000 \\
\hline 3 & $\Delta \mathrm{yf}_{2}(\mathrm{~m})$ & $-10,000$ & 10,000 & $1,668.76$ \\
\hline 4 & $\Delta \mathrm{xf}_{3}(\mathrm{~m})$ & $-10,000$ & 10,000 & 10,000 \\
\hline 5 & $\Delta \mathrm{yf}_{3}(\mathrm{~m})$ & $-10,000$ & 10,000 & 1,409 \\
\hline 6 & $\Delta \mathrm{xf}_{4}(\mathrm{~m})$ & $-10,000$ & 10,000 & 4,111 \\
\hline 7 & $\Delta \mathrm{yf}_{4}(\mathrm{~m})$ & $-10,000$ & 10,000 & 305 \\
\hline 8 & $\mathrm{~V}_{\text {dock }}(\mathrm{m} / \mathrm{s})$ & 3 & 10 & 3 \\
\hline 9 & $\mathrm{nf}_{3 \mathrm{rd}}(\%)$ & 80 & 90 & 89 \\
\hline & & & & \\
\hline
\end{tabular}

Figure 4 presents the trend of the Fitness during the optimization with the PSO algorithm: 30 individuals has been considered, and a number of 20 iterations in positions update have been considered: the PSO algorithm usually works very well in optimization problems in which the Fitness presents a smooth behaviour in function of the change of parameters to be optimized, providing consistent results in a short time. The number of individuals has been set to 
adequately explore the space of solutions, while the number of iterations has been defined after some tests showing no further improvements in solution for values beyond that set in the simulations.

Figure 4 Example of trend of the Fitness during the feeder trajectory optimization.

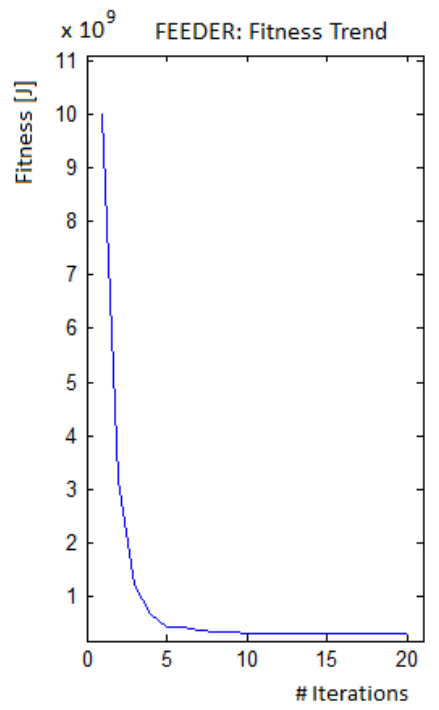

Just to provide some data about the parameters change along the trajectory in the optimized scenario, Figure 5 depicts the longitudinal speed and thrust, while Figure 6 provides the $(B-W) / W$ ratio, and vertical speed along the trajectory for the feeder.

Figure 5 plot of the feeder longitudinal speed (left) and thrust (right)
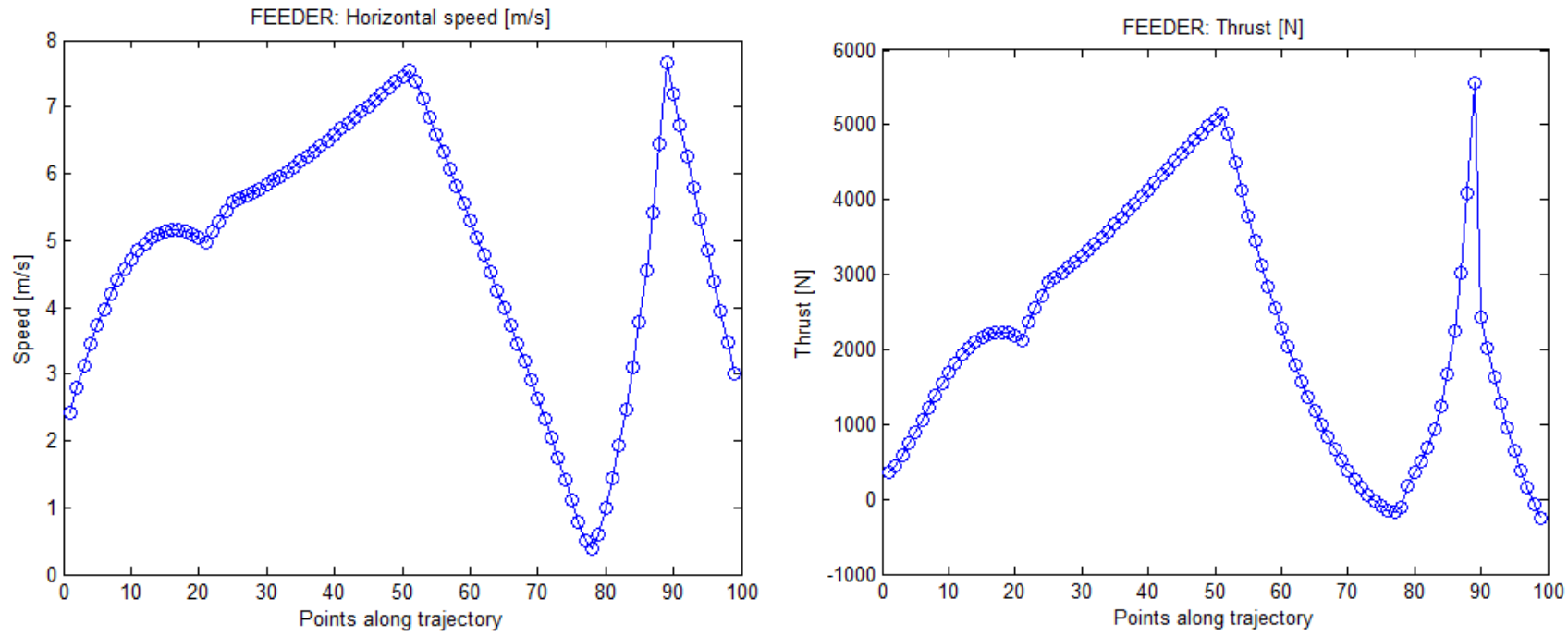

Figure 6 plot of the feeder vertical speed (left) and (B-W)/W term (right) 

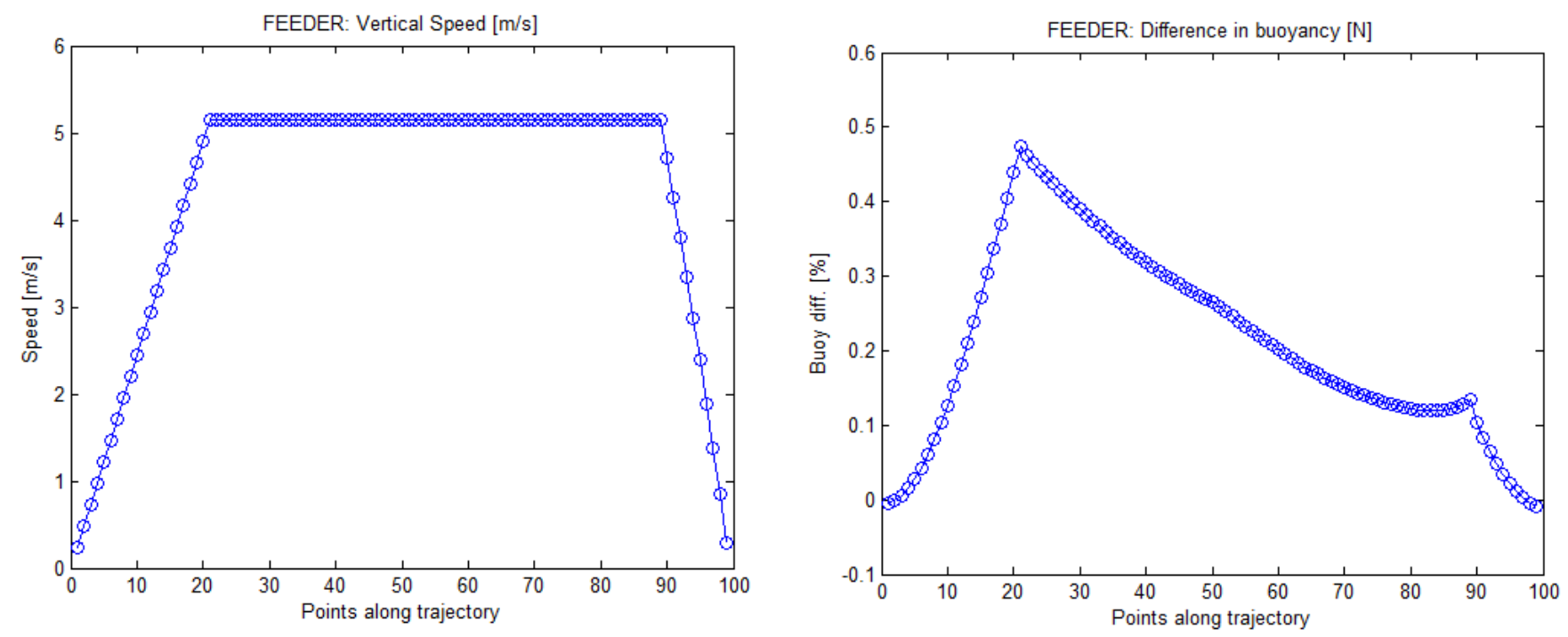

As expected, it is easy to detect the segments in which the trajectory has been divided. The change of the parameters monitored in the figures is quite smooth and it appears that the power system of the feeder is able to provide the thrust and control needed to follow the path.

Table 3 presents the maximum and minimum values of the cruiser trajectory parameters to be optimized, together with the optimized value found after the PSO algorithm application.

Table 3 Parameters to be optimized: cruiser

\begin{tabular}{lllll}
\hline \hline$\#$ & Parameter symbol & Minimum & Maximum & Optimized \\
\hline \hline 1 & $k_{2}(\mathrm{~m})$ & 0.2 & 0.5 & 0.432 \\
\hline 2 & $k_{3}(\mathrm{~m})$ & 1 & 5 & 2.241 \\
\hline 3 & $\Delta \mathrm{xc}_{2}(\mathrm{~m})$ & $-7,500$ & 7,500 & 534.01 \\
\hline 4 & $\Delta \mathrm{yc}_{2}(\mathrm{~m})$ & $-7,500$ & 7,500 & $-7,500$ \\
\hline 5 & $\mathrm{nc} 2$ nd $(\%)$ & 80 & 90 & 80 \\
\hline
\end{tabular}

Also in this case, the trend of the Fitness function along generations is presented in Figure 7; similar settings of the PSO of 30 particles for 25 position updates have been selected.

Figure 7 Example of trend of the Fitness function during the feeder trajectory optimization 


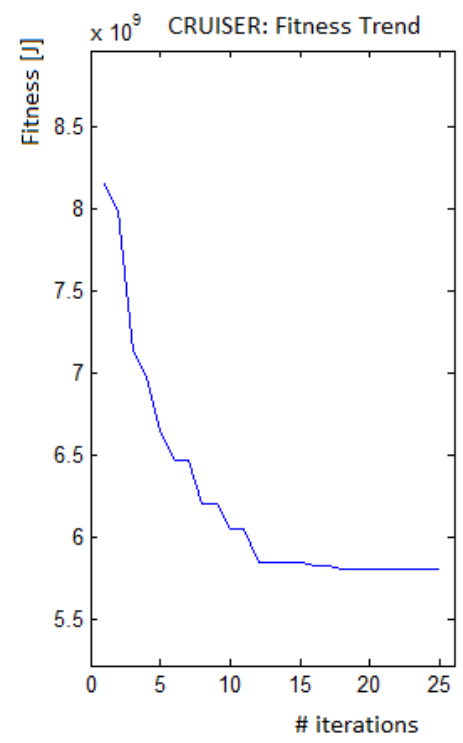

Figure 8 presents on the right the evolution of the longitudinal speed along the track, and on the left the corresponding value of the cruiser longitudinal thrust. As already discussed, the change in velocity from the cruising speed to the docking speed should be on the one hand smooth to reduce the deceleration on the cruiser (see thrust), and on the other hand fast to keep the speed high (controllability). It is quite important to have a good lateral control provided by vertical surfaces giving a lateral force proportional to the square of the speed up to the docking position.

Figure 8 Cruiser longitudinal speed (left) and thrust (right).
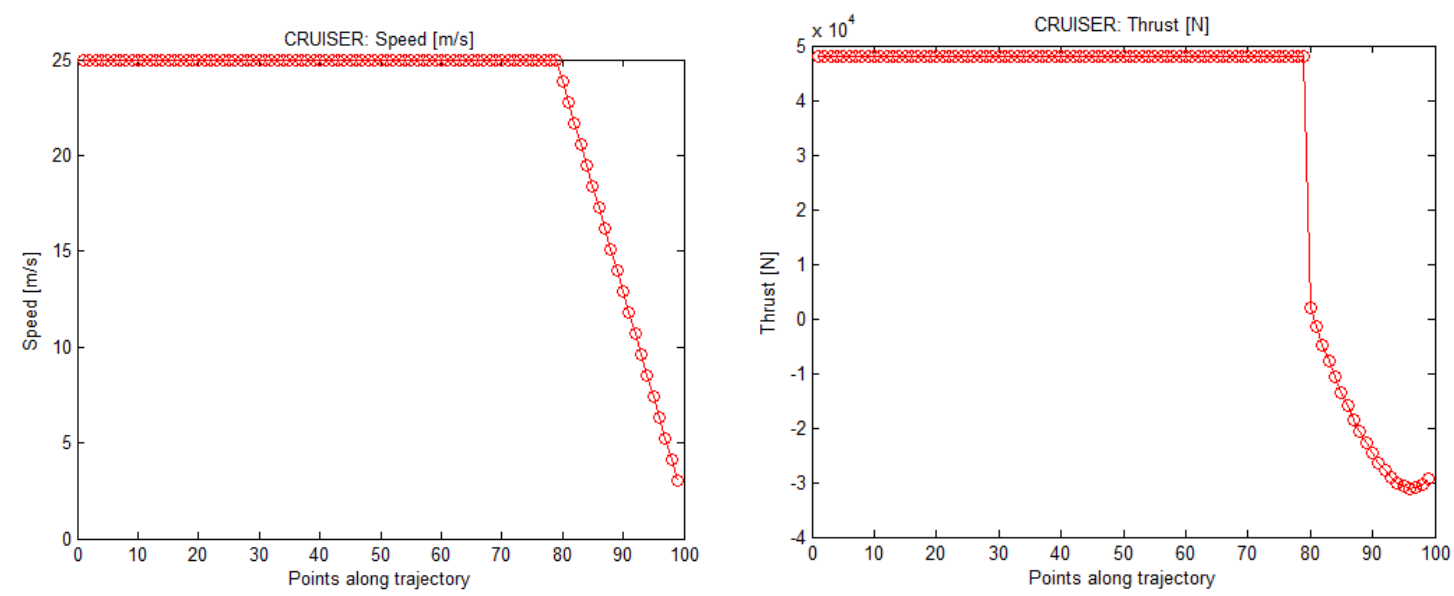

Figure 9 illustrates the value of the lateral force and course of the cruiser along the trajectory. As it can be seen from the images, the lateral force is bounded within values acceptable for the passengers' comfort, while the change in cruiser course is continuous and smooth. Both for cruiser and feeder for all the steps the system of Eqs. 8 and 12 have solution. This implies that the number $N_{u n f}$ is zero in both cases; unitary weights have been used both for $k f f_{1,2}$ and $k f c_{1,3}$, while $k f f_{3}=k f c_{2}=2$. 
Figure 9 Cruiser lateral force (left) and course (right)
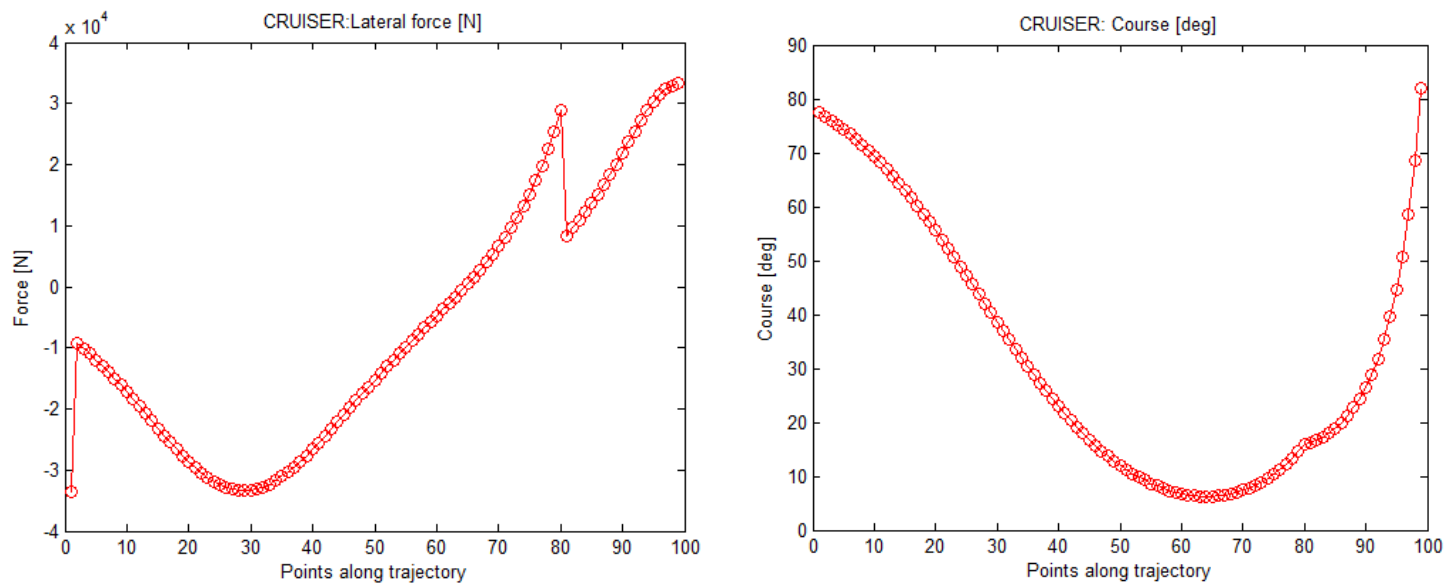

Finally, Figure 10 presents both the trajectory of the feeder and cruiser; it is worth noting that the trajectories are tangent each other at the ending tip, so that the cruiser and feeder presents at the docking point same speed and course. The five control points of the Bezier Curves representing the Cruiser and Feeder trajectories are shown in Figure 10, while the single points along the trajectories are not displayed to increase the figure readability. A final vertical manoeuvre is requested for the joining of the two vehicles, but small adjustments are required. This delicate phase is however not detailed in the analysis carried forward. A perfect time coordination between cruiser and feeder is needed to allow the docking. The final energy required along the trajectories is equal to $1991.3 \mathrm{MJ}$ for the cruiser, and $49.6 \mathrm{MJ}$ for the feeder.

Figure 10 Cruiser and feeder trajectories; 3d (left) and 2d (right)
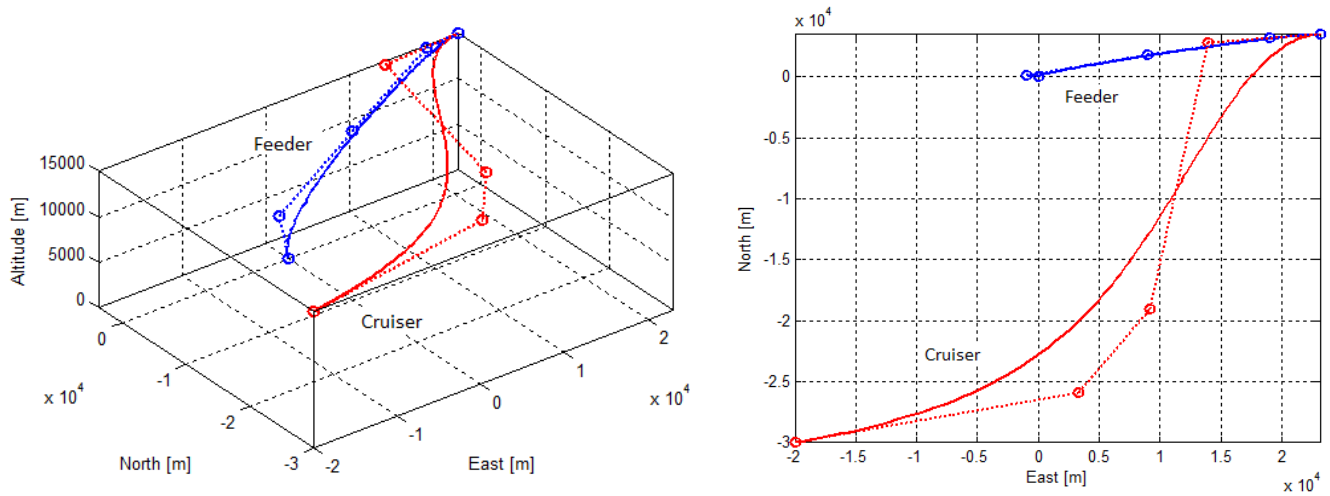

\section{Conclusion}

Aim of this paper is to present an appropriate strategy to study the docking between unconventional airships at altitude.

The trajectory of a cruiser flying at altitude and a feeder airship lifting from ground up are studied. The course of the airships have been modelled through Bezier curves and optimized in order to respect the constraints due to the power 
and performances of the two airships. Following a set of initial tests, not included for sake of brevity, it is possible to comment the problem of the docking. When an unconventional configuration using a feeder similar to a hot-air balloon is selected it is impossible to control with high level of accuracy the ascent of the airship. One good strategy could be to partially withstand the effect of the wind in the first part of the trajectory, and control the airship in the final part of the path to reach a useful docking speed. With such a feeder airship the most demanding part of the docking is requested to the cruiser airship whose trajectory should meet the feeder with the same speed and course. The use of a more streamlined conventional feeder airship (with a top speed larger by far than the cruiser) would help the docking problem, allowing the cruise to follow its original track and letting the alignment manoeuvre to be made by the feeder. The problem of the docking requires a perfect knowledge of the atmospheric wind, whose effect should be kept into account for the docking. In case of stronger wind condition, the docking with unconventional feeders requires large thrusts, to increase manoeuvrability. It can happen also that the cruiser speed is too low to assure a docking manoeuvre; it is in fact important to note that the lateral/directional dynamics of an airship is highly dependent on its speed, due to the contribution of the vertical control surfaces. The mathematical framework herein implemented could be implemented in a control system to support the airship Flight Management System in the automatic docking, provided an accurate wind forecast is available.

\section{Further Work}

As suggested by Lee et al. (2014) a 6 DOF model would provide more accurate results and a perfect matching between trajectory optimization and nonlinear tracking controls. As also indicated by these authors, when considering the moments acting on the airships, the effect of the change in the Centre of Buoyancy with altitude could provide accurate results and the detailed design of controls and power systems can start. The effect of wind gusts should also be considered, since it is expected that will affect the thrust needed to follow the trajectory and its shape: the Dryden model could be implemented to this aim, although it is likely that a high number of division steps along the trajectory would be required to capture the effect of the wind gusts on the cruiser and feeder trajectories.

\section{Acknowledgments}

This research has been supported by the European Union, Seventh Framework Programme (FP7/2007-2013), under grant agreement n FP7-AAT GA-2011-285602-MAATMultibody Advanced Airship for Transport.

\section{References}

Atashpaz-Gargari, E., and C. Lucas, C. (2007), Imperialist Competitive Algorithm for Optimization Inspired by Imperialistic Competition, IEEE Congress in Evolutionary Computation, Singapore, 2007.

Betts, J. (1998), Survey of numerical methods for trajectory optimization, Journal of Guidance, Control, and Dynamics, Vol. 21, No. 2, 1998, pp. 193-207. 
Blasi, L., Barbato, S., and Mattei, M. (2013), A particle swarm approach for flight path optimization in a constrained environment, Aerospace Science and Technology 26 (2013) pp. 128-137.

Carichner, G.E., and Nicolai, L. 2013, Fundamentals of Aircraft and Airship Design, Volume Two - Airship Design and Case Studies, AIAA Education Series, April 2013, ISBN-13: 978-1600868986.

Ceruti, A. and Marzocca, P. (2014), Conceptual Approach to Unconventional Airship Design and Synthesis, J. Aerosp. Eng., 27(6), DOI: 10.1061/(ASCE)AS.1943-5525.0000344.

Ceruti, A., Voloshin, V., and Marzocca, P. (2014), Heuristic Algorithms Applied to Multidisciplinary Design Optimization of Unconventional Airship Configuration, AIAA Journal of Aircraft, DOI: 10.2514/1.C032439.

Chen, Q., Zhu, M., and Sun, K. (2011), Analysis to Effects on Conceptual Parameters of Stratospheric Airship with Specified Factors, Journal of Computers, Vol. 6, No.5, 2011, pp. 1055-1062.

Duan, H., Liu S., and Lei, X. (2008), Air robot path planning based on Intelligent Water Drops optimization, IEEE International Joint Conference on Neural Networks IJCNN 2008, pp. 1397 - 1401.

Dumas A., Trancossi M., Madonia M., and Giuliani I. (2011), Multibody advanced airship for transport, SAE International, DOI: 10.4271/2011-01-2786.

Ellenrieder T. J. (1999), The modelling and validation of the vertical dynamics of a hot air balloon, Proc Instn Mech Engrs Vol 213 Part G.

Farin, G. (1990), Curves and Surfaces for Computer Aided Geometric Design, ed. Academic Press, San Diego, CA (USA) 1990.

Fishman, G.S. (1995), Monte Carlo: Concepts, Algorithms, and Applications, Springer, New York, 1995, ISBN 0-38794527-X.

Gil, P.E., Murray, W., and Saunders, M.A., (2005), SNOPT: An SQP Algorithm for Large-Scale Constrained Optimization, SIAM Review, Vol. 47. N0 1, pp 99-131.

Goldberg, D. E. (1989), Genetic Algorithms in Search, Optimization and Machine Learning, Addison-Wesley Pub. Co, Reading, MA, 1989.

Guo, X., and Zhu, M. (2013), Ascent trajectory optimization for stratospheric airship with thermal effects, Advances in Space Research 52:6, 2013, pp. 1097-1110.

Hsiao Y., Chuang C.L., Jiang, J.A., and Chien, C.C. (2005), A Novel Optimization Algorithm: Space Gravitational Optimization, Proc. of 2005 IEEE international conference on Systems, Man and Cybernetics, vol.3, pp. 2323-2328.

Ilieva, G., Páscoa, J., Dumas, A., and Trancossi, M. (2014), MAAT - Promising innovative design and green propulsive concept for future airship's transport, Aerospace Science and Technology, Volume 35, May 2014, Pages 114, ISSN 1270-9638, http://dx.doi.org/10.1016/j.ast.2014.01.014.

Kennedy, J., and Eberhart, R.C. (1995), Particle swarm optimization, Proceedings of IEEE international conference on neural networks, vol.4, 1995, pp. 1942-1948.

Khoury, G.A., and Gillet, J.D. (1999), Airship Technology, Cambridge University Press, Cambridge, UK.

Lee, S., Jang, J. Ryu, H., and Lee, K.H. (2014), Matching trajectory optimization and nonlinear tracking control for HALE, Advances in Space Research 54, pp.1870-1887.

Liao, L., and Pasternak, I. (2009), A review of airship structural research and development, Progress in Aerospace Sciences, Vol. 45, No. 4-5, 2009, pp. 83-96.

Mueller, J.B. (2013), Design and Analysis of Optimal Ascent Trajectories for Stratospheric Airships, PhD Thesis, August 2013, University of Minnesota, available on line at: http://conservancy.umn.edu/bitstream/158923/1/Mueller_umn_0130E_14295.pdf 
Mueller, J., Zhao, Y., and Garrard, W. (2009a), Sensitivity and Solar Power Analysis of Optimal Trajectories for Autonomous Airships, AIAA Guidance, Navigation, and Control Conference 10 - 13 August 2009, Chicago, Illinois.

Mueller, J. B., Zhao, Y. J., and Garrard, W. L. (2009b), Optimal Ascent Trajectories for Stratospheric Airships, Journal of Guidance, Control, and Dynamics, Vol. 32, No. 4, July/Aug 2009, pp. 1232-1245.

Murray, W., Gill, P. E., and Saunders, M. A. (2002), SNOPT: An SQP Algorithm for Large-Scale Constrained Optimization, SIAM Journal of Optimization, Vol. 12, 2002, pp. 979-1006.

Mortenson, M.E. (1999), Mathematics for Computer Graphics Applications: An Introduction to the Mathematics and Geometry of CAD/Cam, Geometric Modelling, Scientific Visualization, 2nd edition, Industrial Press, Inc. New York, NY, USA @1999, ISBN:083113111X.

Rademacker, A.T. (2014), Very Large Luxury Airship (VLLA), American Institute of Aeronautics and Astronautics, http://www.engr.sjsu.edu/nikos/pdf/VLLAirship. Available on line, accessed on August 2014.

Smith, T., Bingham, C., Stewart P., Allarton, R., and Stewart, J. (2013), Energy harvesting and power network architectures for the multibody advanced airship for transport high altitude cruiser-feeder airship concept, Proceedings of the Institution of Mechanical Engineers, Part G: Journal of Aerospace Engineering, April 2013 vol. 227 no. 4 pp. 586-598.

Stockbridge C., Ceruti A., Marzocca P. (2013), Airship Research and Development in the Areas of Design, Structures, Dynamics and Energy Systems, International Journal Aeronautical and Space Sciences Vol. 13, no. 2, 2012, pp. 170187.

Storn, R., and Price, K. (1997), Differential evolution - a simple and efficient heuristic for global optimization over continuous spaces, Journal of Global Optimization, vol. 11,pp. 341-359.

Tornabene, F., and Ceruti, A. (2013), Mixed Static and Dynamic Optimization of Four-Parameter Functionally Graded Completely Doubly Curved and Degenerate Shells and Panels Using GDQ Method, Mathematical Problems in Engineering, Vol. 2013, Article ID 867079, pp 1-33, http://dx.doi.org/10.1155/2013/867079.

Tuveri, M., Ceruti, A., Marzocca, P. (2014), Added masses computation for unconventional airships and aerostats through geometric shape evaluation and meshing, Int'l J. of Aeronautical \& Space Sci. 15(3), pp. 241-257 DOI:10.5139/IJASS.2014.15.3.241.

Yang, X. S. (2005), Biology-derived algorithms in engineering optimizaton (Chapter 32), in Handbook of Bioinspired Algorithms and Applications (eds Olarius \& Zomaya), Chapman \& Hall /CRC.

Yu, D., and Lu, X. (2010), Configurations analysis for high altitude/long-endurance airships, Aircraft Engineering and Aerospace Technology, Vol. 82, No. 1, pp. 48-59.

Yuwen, L., Nahon, M., and Sharf, I. (2011), Airship dynamics modelling: A literature review, Progress in Aerospace Sciences, Vol. 47, No. 3, 2011, pp. 217-239.

\section{Nomenclature}

\section{Symbols}

$A_{\text {lat }}$ - lateral plane projection of the airship shape $\left[\mathrm{m}^{2}\right]$

$A_{v e r t}$ - horizontal plane projection of the airship shape

$\left[\mathrm{m}^{2}\right]$ $b_{i, n}(t)$ - Bernstein basis polynomial of degree $n[-]$

$B$ - airship buoyancy $[\mathrm{N}]$

$B(t)$ - column vector with 3D Bezier curve points [-] 
$D$ - airship drag $[\mathrm{N}]$

$F_{\text {lat }}$ - lateral force $[\mathrm{N}]$

$L$ - airship lift $[\mathrm{N}]$

Lat, Long, $h$ - Latitude, Longitude and Altitude [rad, rad,

$\mathrm{m}]$

$M$ - airship mass $[\mathrm{kg}]$

$M_{a x L T}$ - maximum allowable lateral thrust $[\mathrm{N}]$

$m_{a x}, m_{a y}, m_{a z}$ - airship added masses in longitudinal, lateral and vertical direction $[\mathrm{kg}]$

$n$ - degree of the Bezier curve [-]

$N$ - number of points in which the trajectory is divided [-]

NC2 - point of the cruiser trajectory in which the second segment starts.

$n c_{2 n d}-\%$ of the total number of points of the cruiser trajectory corresponding to NC2

NF3 - point of the feeder trajectory in which the third segment starts.

$n f_{3 r d}-\%$ of the total number of points of the feeder trajectory corresponding to NF3.

$N_{u n f}-$ number of steps in which the system of equation 8 (for cruiser) or 12 (for feeder) has no solution.

$P_{i}$ - column vector with coordinates of the Bezier curve $i$ th control point [m]

$P c_{i}, P f_{i}$ - coordinates of the cruiser and feeder airships BC based trajectory control points [m]

$R$ - Earth mean Radius [m]

Space $_{i}$ - space covered by the feeder and cruiser in a step, equal to the distance between two consecutive points in the trajectory.

$t$ - parameter crossing along the $\mathrm{BC}[0 \div 1]$
$T$ - airship thrust [N]

$T_{\max }-$ maximum allowable longitudinal thrust [N]

$V$ - airship speed along the trajectory $[\mathrm{m} / \mathrm{s}]$

Vol - airship volume $\left[\mathrm{m}^{3}\right]$

$W$ - airship weight $[\mathrm{N}]$

$W_{\text {East }}, W_{\text {North }}, W_{u p}$ - components of the wind speed along the North, East and Up direction [m/s]

$W_{x}, W_{y}, W_{z}$ - projection of the Wind speed along the airship body axis $[\mathrm{m} / \mathrm{s}]$

$k_{1}$ - parameter to define the $2^{\text {nd }}$ control point of the feeder

$\mathrm{BC}$ based trajectory [-]

$k_{2}, k_{3}$ - parameter to define $2^{\text {nd }}$ and $4^{\text {th }}$ control point of the cruiser BC based trajectory [-]

$k f f_{1,2,3}$ - weight coefficients for the feeder Fitness

$k f c_{1,2,3}-$ weight coefficients for the cruiser Fitness

$Z_{\text {dock }}$ - docking altitude [m]

$\alpha$ - angle of attack (AoA) [rad]

$\Delta x f_{i}, \Delta y f_{i}-x$ and $y$ delta position of the control points of the $\mathrm{BC}$ for feeder trajectory [m]

$\Delta x c_{2}, \Delta y c_{2}-\mathrm{x}$ and $\mathrm{y}$ delta position of the control points of the BC for cruiser trajectory [m]

$\Delta t$ - time required to cover the distance between two consecutive trajectory points [s]

$\Delta X, \Delta Y, \Delta Z$ - difference between the position of two consecutive trajectory points [m]

$\mathrm{Y}$ - trajectory angle [rad]

$\mu$ - angle between airship reference line and the propulsion system thrust [rad]

$\rho$ - air density at current altitude $\left[\mathrm{kg} / \mathrm{m}^{3}\right]$

$\psi$ - course angle respect to the North [rad] 
Definitions, Acronyms and Abbreviations

AM: Added Masses

AoA: Angle of Attack

BC: Bezier Curve 